\title{
Multi-frequency long-term observations of Her X-1 - The 35-d cycle
}

N. Shakura*, ${ }^{1,2}$ D. Kolesnikov, ${ }^{1}$ K. Postnov, ${ }^{1,2}$ I. Volkov, ${ }^{1,3}$ I. Bikmaev, ${ }^{2}$

T. Irsmambetova, ${ }^{1}$ R. Staubert, ${ }^{4}$ J. Wilms, ${ }^{5}$ E. Irtuganov, ${ }^{2}$ P. Shurygin, ${ }^{2}$ P. Golysheva, ${ }^{1}$ S. Shugarov, ${ }^{1,6}$ I. Nikolenko, ${ }^{3,7}$ E. Trunkovsky, ${ }^{1}$ G. Schonherr, ${ }^{8}$ A. Schwope,${ }^{8}$ D. Klochkov ${ }^{4}$

${ }^{1}$ Sternberg Astronomical Institute, Moscow State University, Moscow, Russia

${ }^{2}$ Kazan Federal University, Kazan, Russia

${ }^{3}$ Institute of Astronomy RAS, Moscow, Russia

${ }^{4}$ Institute for Astronomy and Astrophysics, Tubingen, Germany

${ }^{5}$ Astronomical Institute of the University of Erlangen-Nuremberg, Bamberg, Germany

${ }^{6}$ Astronomical Institute of the Slovak Academy of Scienses, Tatranska Lomnica, Slovakia

${ }^{7}$ Crimean Astrophysical Observatory, Nauchny

${ }^{8}$ Leibniz Institute for Astrophysics, Potsdam, Germany

In this work are presented the results of modelling of $35^{d}$ superorbital changes of $B$ and $V$ lightcurves and X-ray flux of HZ Her/Her X-1. The model implemented in the new code written in $\mathrm{C}$ programming language, with module for parameter optimisation written in Python. The model includes a tilted precessing and warped accretion disc around a freely precessing neutron star. The disc is warped near its inner edge due to interaction with the rotating neutron star magnetosphere. The magnetic torque depends on the precessional phase of the neutron star. The X-ray emission flux from the neutron star also depends on the free precession phase which modulates the X-ray illumination of the optical star atmosphere and the intensity of gas streams. We demonstrate that this model is able to well reproduce both optical observations of $\mathrm{HZ} \mathrm{Her} \mathrm{and} \mathrm{the} \mathrm{behaviour} \mathrm{of} \mathrm{the}$ 35-day X-ray cycle

Multifrequency Behaviour of High Energy Cosmic Sources - XIII - MULTIF2019

3-8 June 2019

Palermo, Italy

${ }^{*}$ Speaker. 


\section{Introduction}

$\mathrm{HZ} \mathrm{Her} / \mathrm{Her} \mathrm{X}-1$ is an intermediate mass X-ray binary consisting of a $1.8-2.0 M_{\odot}$ evolved sub-giant star and an 1.0-1.5 $M_{\odot}$ neutron star observed as X-ray pulsar [1]. The binary orbital period is $P_{b}=1.7$ days, the X-ray pulsar spin period is $P_{*}=1.24$ seconds. The optical star fills its Roche lobe and an accretion disk is formed around the neutron star (Fig. 1). Due to X-ray irradiation, the optical flux from HZ Her is strongly modulated with the orbital period, as was first found by the inspection of archive photoplates [2]. Before X-ray observations, HZ Her had been classified as an irregular variable.

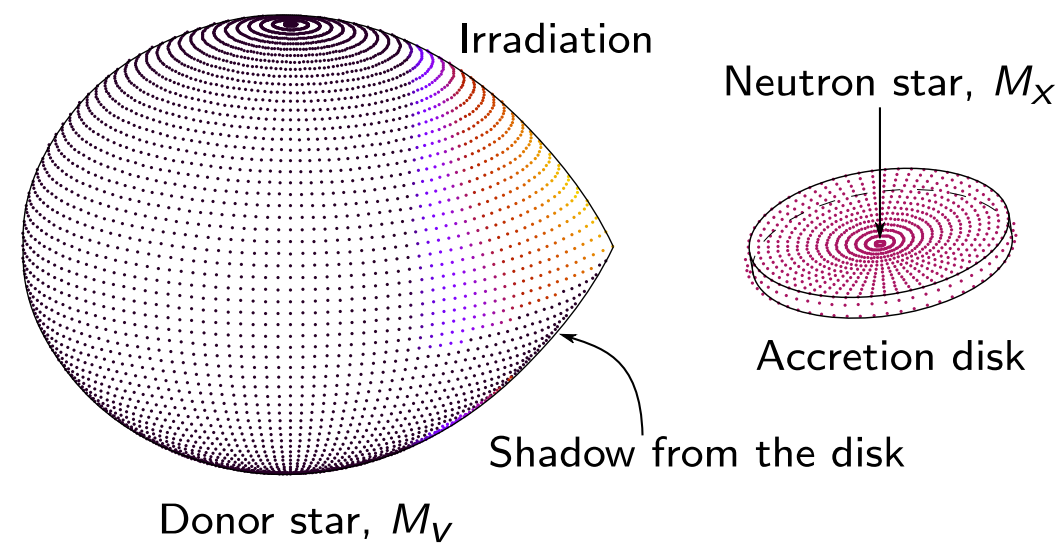

Figure 1: Appearance of the HZ Her/Her X-1. The binary orbital period is $P_{b}=1.7^{d}$, the X-ray pulsar spin period is $P_{*}=1.24^{s}$. The donor star fills its Roche lobe and an accretion disk is formed around the neutron star. Due to X-ray irradiation, the optical flux from HZ Her is strongly modulated with the orbital period.

The X-ray flux curve of Her X-1 is modulated with $\approx 35$ day period. The $35^{d}$-day X-ray cycle consists of a 7-orbit "main-on" state and a 5-orbit "short-on" state of lower intensity (Fig 2). The main-on and short-on states are separated by 4-orbit intervals during which the X-ray flux vanishes completely. The $\mathrm{X}$-ray observations can be explained by eclipses of the central source due to the motion of a precessing accretion disk [3]. Also in the last work and in [4] were performed pioneering works for modelling of the superorbiral $35^{d}$-period changes of lightcurves.

Soon after the discovery of the X-ray pulsar, the NS free precession was suggested as a possible explanation to the observed $35^{d}$-day modulation [6]. Later on, the EXOSAT observations of the evolution of X-ray pulse profiles of Her X-1 with the 35-day cycle phase was also interpreted by the NS free precession [7]. Another model, based on the precession of the accretion disk, have been first presented in the paper of [8].

Extensive studies of Her X-1 suggested the presence of a warped tilted accretion disk around NS. Its retrograde precession results in consecutive opening and screening of the central X-ray source [9]. The X-ray light curve is asymmetric between the eclipses due to scattering of X-ray radiation in a hot rarefied corona above the disk. Indeed, the X-ray "turn-on" at the beginning of the "main-on" state is accompanied by a significant decrease in the soft X-ray flux because of strong absorption. No essential spectral change during the X-ray flux decrease is observed, suggesting the photon scattering on free electrons of the hot corona close to the disk inner edge [10, 11, 12, 13]. 

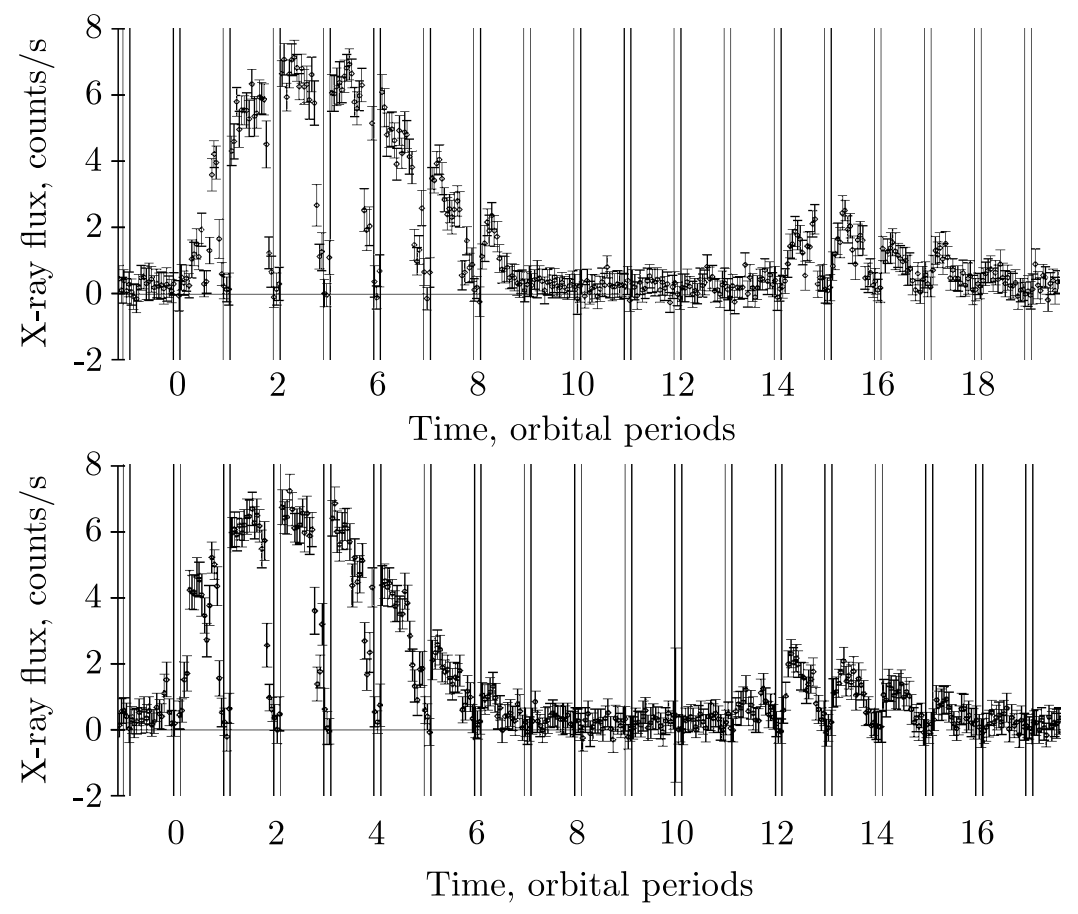

Figure 2: $35^{d}$ cycle of X-ray flux of Her X-1. The $35^{d} \mathrm{X}$-ray cycle consists of a 7-orbit "main-on" state and a 5-orbit "short-on" state of lower intensity. The $35^{d}$ cycle of the Her X-1 is explained by the accretion disk precession in the direction opposite to the orbital motion. Top figure show X-ray flux with the start of the main-on at orbital phase 0.7 , bottom one with the start of the main-on at orbital phase 0.2. Figure from [5].

The X-ray pulse profile are observed to strongly vary with the $35^{d}$-day phase $[7,14,15,16]$ differing significantly at the main turn-on and of the short-on states. Such changes are difficult to explain by the precessing disk only. As was shown by [17], the X-ray RXTE/PCA pulse evolution with the $35^{d}$ phase can be explained by the NS free precession with a complex magnetic field structure on the NS surface. In this model, in addition to the canonical poles (a dipole magnetic field), arc-like magnetic regions around the magnetic poles are included, which is a consequence of a likely non-dipole surface magnetic field of the neutron star [18, 19].

Longterm observations show that, there was long (up to 1.5 years) off-states of X-ray sourse, but X-ray irradiation remains unchanged [20, 21, 22, 23, 24]. Possibly, that mean accretion disk had layed in the orbital plane and X-ray source was obscured to observer. Till now it is not clearly known which mechanism take disk out of orbital plane and put it back.

History of the optical observations of HZ Her written on glass photoplates, show us long periods of absense of X-ray irradiation [25, 26]. It means in that periods accretion completely vanishes.

In the present work, we have analyzed the nature of $35^{d}$ cycle on the base of an extensive optical photometric observations of HZ Her collected from the literature and obtained by the authors. We have found that the the model of precessing tilted and warped accretion disk combined with the free precession of the neutron star with complex surface magnetic field is able to explain a detailed photometric light curve of HZ Her constructed from all observations available. 


\section{Model}

Donor star shape is defined by equipotential surface of the Roche potential. The effective temperature of the donor star varies across the surface due to gravity darkening and X-ray irradiation. The disk in the model warped and tilted (Fig. 3). The disk makes a complex X-ray shadow on the surface of the donor star. We suggest that the disk warped mostly near the neutron star due to magnetic torque. Near the orbital phase 0.5 disk passes in front of the donor star. We use a

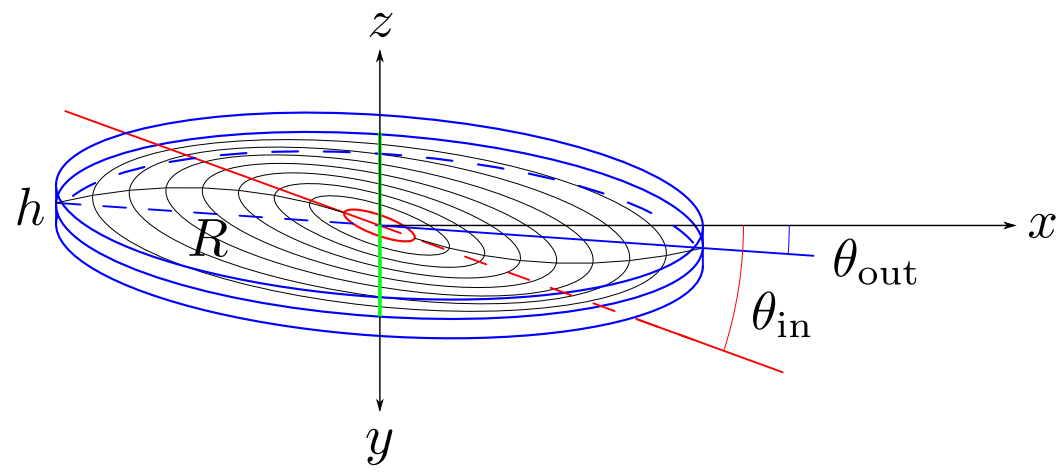

Figure 3: Model of the disk. There are 7 free parameters in the model that completely define the shape and orientation of the disk: $\theta_{\text {out }}$ - tilt of the outer edge of the disk with respect to the orbital plane, $\theta_{\text {in }}$ - tilt of the inner edge with respect to the orbital plane, $\varphi_{\text {out }}$ - phase angle of precession motion, $Z-$ angle between the nodal line of the outer edge of the disk and the nodal line of the inner edge of the disk (on the figure $Z=0$, see green line), inclination $i$ and phase where $\varphi_{\text {out }}=0$. Phase $\varphi_{\text {out }}=0$ corresponds to the maximum opening of the disk. In addition, the width $h$ of the outer edge of the disk was included in the model.

ray-tracing technique to determine which parts of the star's surface are obscured by the disk. We haven't modelled orbital phases near 0.0, where the disk passes behind the donor star. The neutron star's X-ray intensity adopted from [17]. The neutron star is under free precession with a period close to $35^{d}$.

\section{Results}

Here we show found parameters of the model as a function of the phase of $35^{d}$ cycle. Also we show the lightcurves corresponding to the minimum of residuals between model and observations (Fig. 4). The most interesting result that the the angle $Z$, which defines torsion of the disk, follows the theoretical magnetic torque $K_{m}$ acting on the disk's inner edge (Fig. 6, Z). Inner edge of the disk tends to be close to the NS's equator when magnetic torque reaches maximal value (Fig. 6, $\left.\theta_{i n}\right)$, which was predicted by [27].

Resent XMM-Newton satellite observations showed- the presence of the modulation of disk wind mass outflow rate with $35^{d}$ superorbital period [28]. This result is in according to the model of free precession of the neutron star. Wind becomes stronger when the magnetic pole of the neutron star gets the most close to the disk plane. 


\section{References}

[1] H. Tananbaum, H. Gursky, E. M. Kellogg, R. Levinson, E. Schreier and R. Giacconi, Discovery of a Periodic Pulsating Binary X-Ray Source in Hercules from UHURU, ApJ 174 (1972) L143.

[2] A. M. Cherepashchuk, Y. N. Efremov, N. E. Kurochkin, N. I. Shakura and R. A. Sunyaev, On the Nature of the Optical Variations of HZ Her = Her X1, Information Bulletin on Variable Stars $\mathbf{7 2 0}$ (1972).

[3] D. Gerend and P. E. Boynton, Optical clues to the nature of Hercules X-1/HZ Herculis, ApJ 209 (1976) 562.

[4] I. D. Howarth and B. Wilson, A Study of the Low-Mass X-Ray Binary Hz-Herculis / HERCULES-X-1 Using IUE and Optical Data, MNRAS 202 (1983) 347.

[5] D. K. Klochkov, N. I. Shakura, K. A. Postnov, R. Staubert, J. Wilms and N. A. Ketsaris, Observational manifestations of the change in the tilt of the accretion disk to the orbital plane in her $x-1 / h z$ her with phase of its 35-day period, Astronomy Letters 32 (2006) 804.

[6] K. Brecher, Her X-1: A Processing Binary Pulsar?, Nature 239 (1972) 325.

[7] J. Truemper, P. Kahabka, H. Oegelman, W. Pietsch and W. Voges, EXOSAT observations of the 35 day cycle of Hercules X-1 Evidence for neutron star precession, ApJ 300 (1986) L63.

[8] E. K. Sheffer, I. F. Kopaeva, M. B. Averintsev, G. S. Bisnovatyi-Kogan, I. M. Golynskaya, L. S. Gurin et al., X-Ray Studies of the HERCULES-X-1 Pulsar with the Astron Satellite, Soviet Ast. 36 (1992) 41.

[9] R. Giacconi and R. Ruffini, eds., Physics and astrophysics of neutron stars and black holes, 1978.

[10] R. H. Becker, E. A. Boldt, S. S. Holt, S. H. Pravdo, R. E. Rothschild, P. J. Serlemitsos et al., Spectra of Hercules X-1 near a turn-on in the 35 day cycle, ApJ 214 (1977) 879.

[11] P. J. N. Davison and A. C. Fabian, Copernicus and Ariel V observations of Hercules X-1, MNRAS 178 (1977) 1P.

[12] A. N. Parmar, P. W. Sanford and A. C. Fabian, Further Ariel V observations of Hercules X-1, MNRAS 192 (1980) 311.

[13] M. Kuster, J. Wilms, R. Staubert, W. A. Heindl, R. E. Rothschild, N. I. Shakura et al., Probing the outer edge of an accretion disk: a Her X-1 turn-on observed with RXTE, A\&A 443 (2005) 753 [astro-ph/0507198].

[14] J. E. Deeter, D. M. Scott, P. E. Boynton, S. Miyamoto, S. Kitamoto, S. Takahama et al., The 35 Day Evolution of the Hercules X-1 Pulse Profile: GINGA Observations and Their Implications, ApJ 502 (1998) 802.

[15] D. M. Scott, D. A. Leahy and R. B. Wilson, The 35 Day Evolution of the Hercules X-1 Pulse Profile: Evidence for a Resolved Inner Disk Occultation of the Neutron Star, ApJ 539 (2000) 392 [astro-ph/0002327].

[16] R. Staubert, D. Klochkov, D. Vasco, K. Postnov, N. Shakura, J. Wilms et al., VizieR Online Data Catalog: Her X-1 pulse profiles (Staubert+, 2013), VizieR Online Data Catalog 355 (2013).

[17] K. Postnov, N. Shakura, R. Staubert, A. Kochetkova, D. Klochkov and J. Wilms, Variable neutron star free precession in Hercules $X-1$ from evolution of RXTE X-ray pulse profiles with phase of the 35-d cycle, MNRAS 435 (2013) 1147 [1307. 6026]. 
[18] N. I. Shakura, K. A. Postnov and M. E. Prokhorov, Presence of a Quadrupole Component of the Magnetic Field Near the Surface of the Neutron Star in HERCULES-X-1, Soviet Astronomy Letters 17 (1991) 339.

[19] I. E. Panchenko and K. A. Postnov, Hercules X-1 pulse profile simulation, A\&A 286 (1994) 497.

[20] A. N. Parmar, W. Pietsch, S. McKechnie, N. E. White, J. Truemper, W. Voges et al., An extended X-ray low state from Hercules X-1, Nature 313 (1985) 119.

[21] S. D. Vrtilek, T. Mihara, F. A. Primini, P. Kahabka, H. Marshall, F. Agerer et al., Multiwavelength Observations of Hercules X-1/HZ Herculis, ApJ 436 (1994) L9.

[22] W. Coburn, W. A. Heindl, J. Wilms, D. E. Gruber, R. Staubert, R. E. Rothschild et al., The 1999 Hercules X-1 Anomalous Low State, ApJ 543 (2000) 351 [astro-ph/9911533].

[23] P. Boyd, M. Still and R. Corbet, Her X-1 Exiting Anomalous Low State, The Astronomer's Telegram 307 (2004) 1.

[24] M. Still and P. Boyd, Fine-Tuning the Accretion Disk Clock in Hercules X-1, ApJ 606 (2004) L135 [astro-ph/0403625].

[25] C. A. Jones, W. Forman and W. Liller, Optical Studies of UHURU Sources.IV. The Long-Term Behavior of HZ HERCULIS=HERCULES X-1, ApJ 182 (1973) L109.

[26] R. Hudec and W. Wenzel, Observations of HZ Her/Her X-1 on Sonneberg Astrograph Plates, Bulletin of the Astronomical Institutes of Czechoslovakia 27 (1976) 325.

[27] V. M. Lipunov, E. S. Semenov and N. I. Shakura, Accretion disk orientation in binary X-ray pulsars, AZh 58 (1981) 765.

[28] P. Kosec, A. C. Fabian, C. Pinto, D. J. Walton, S. Dyda and C. S. Reynolds, An ionised accretion disc wind in Hercules X-1, arXiv e-prints (2019) arXiv:1910.08337 [1910.08337]. 


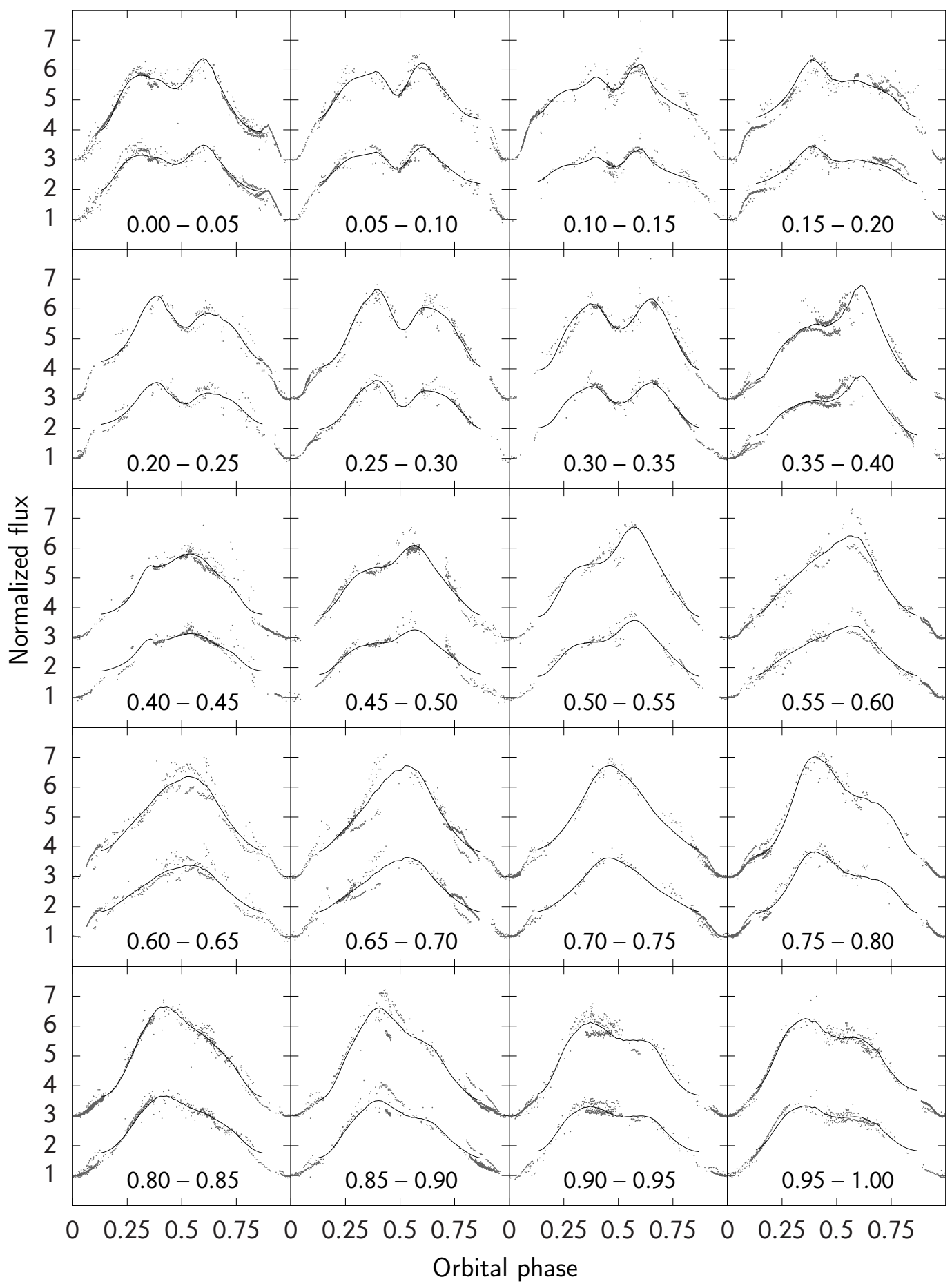

Figure 4: Relative $B$ and $V$ flux. Observed (points) and theoretical lightcurves (solid lines). The numbers in the cells indicate a phase interval of $35^{d}$ cycle. Data in filter $B$ is shifted up to 2 units with respect to $V$ filter. The optimum theoretical lightcurve for every phase is shown. 


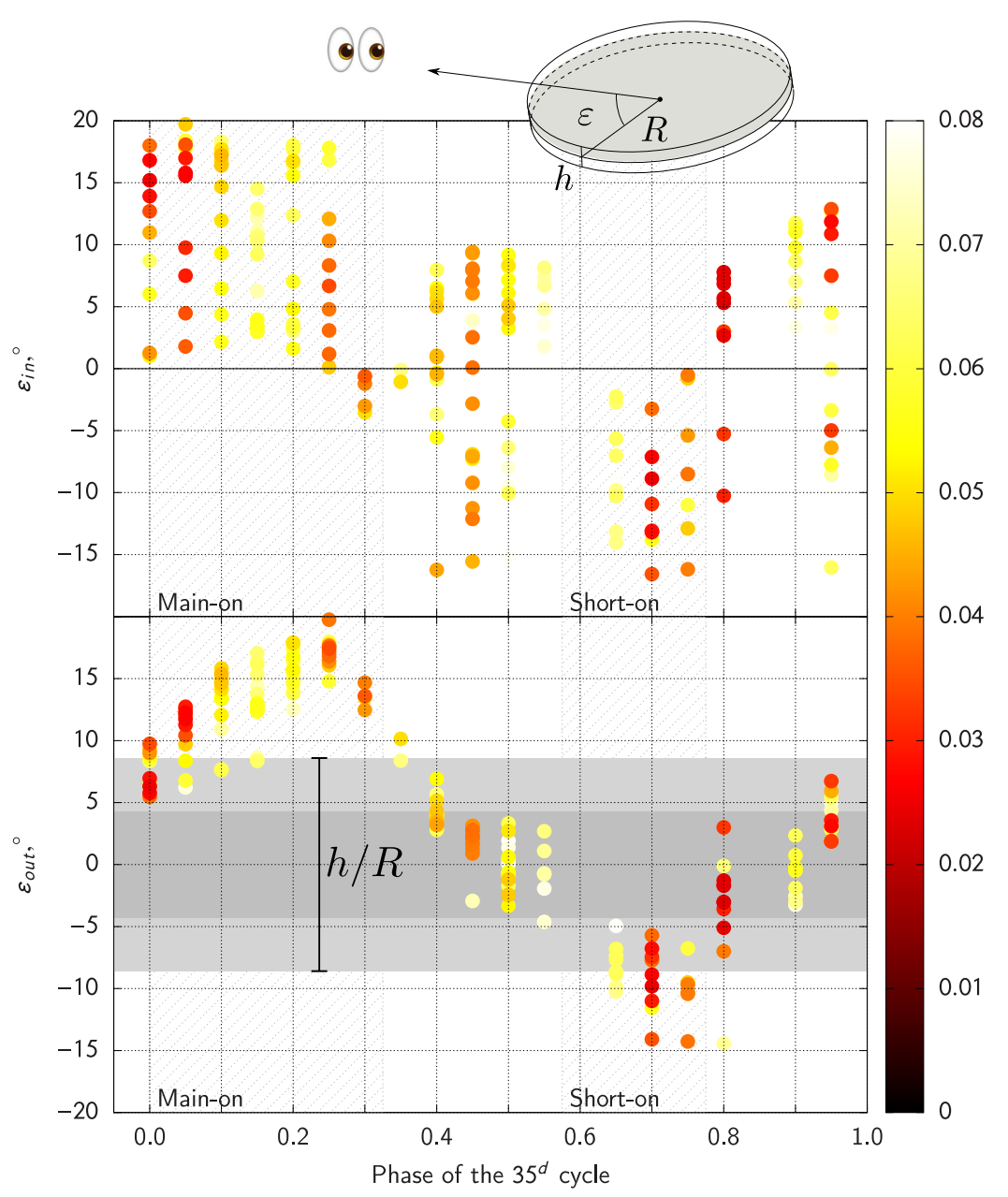

Figure 5: $\varepsilon_{\text {out }}$ is the angle between the observer's line of sight and the disk's outer plane, see picture above graph. $\varepsilon_{i n}$ is the angle between the observer's line of sight and the disk's inner plane. The vertical shaded regions indicate an interval of $35^{d}$ cycle where X-ray flux is visible ("Main-on" and "short-on"). The horizontal shaded area on the second graph is the width of the outer edge of the disk. 


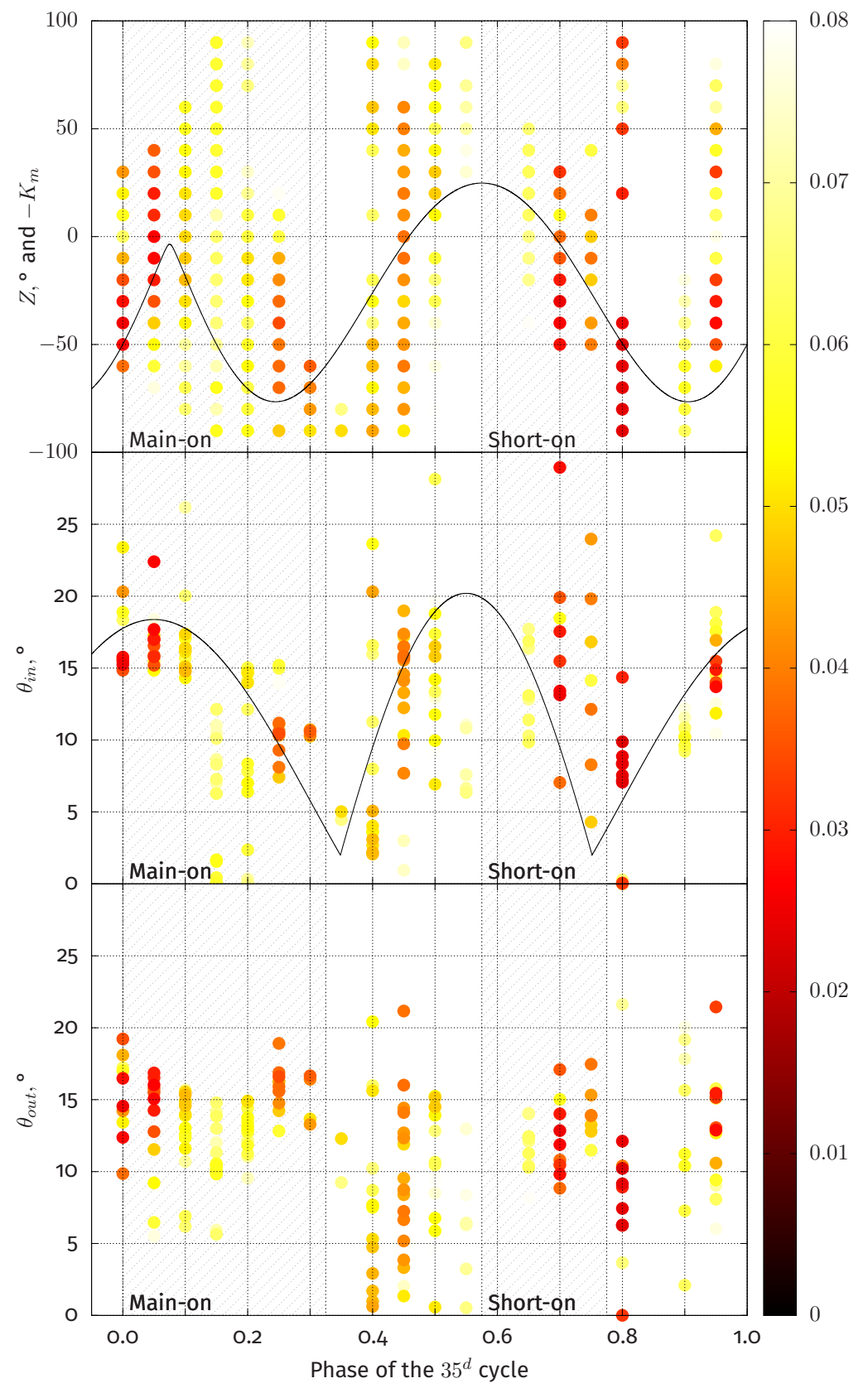

Figure 6: $Z$ is the angle between the nodal lines of the inner and outer edges of the disk. $\theta_{\text {in }}$ and $\theta_{\text {out }}$ are the angles between the disk's plane and the orbital plane (see Fig.3). The solid line on the first graph is the theoretical magnetic torque $K_{m}$ acting on the disk's inner edge [27]. Notice strong corellation of the $\theta_{i n}$ with $Z$. When the magnetic torque $K_{m}$ is maximal, inner edge of the disk becomes closer to the equatorial plane of the neutron star. Change of $Z$ and $\theta_{i n}$ with $35^{d}$ cycle favors to the presence of the free precession of the neuthon star. 